\title{
MYD88 Inhibitor ST2825 Suppresses the Growth of Lymphoma and Leukaemia Cells
}

\author{
ERIKA SHIRATORI, MAI ITOH and SHUJI TOHDA
}

Department of Laboratory Medicine, Tokyo Medical and Dental University, Tokyo, Japan

\begin{abstract}
Background/Aim: Myeloid differentiation primary response gene 88 (MYD88), which activates the nuclear factor kappa $B(N F-k B)$ pathway, is important for the growth of lymphoma and leukaemia cells. In this study, we investigated the effects of ST2825, a synthetic peptidomimetic compound which inhibits MYD88 homodimerization, on their growth. Materials and Methods: Seven lymphoma and leukaemia cell lines including TMD8, a B-cell lymphoma line with MYD88activating mutation, were treated with ST2825 and analysed for cell proliferation and expression of $N F-k B$ signallingrelated molecules. Results: ST2825 suppressed the growth of all cell lines by inducing apoptosis and down-regulating phosphorylation of $N F-k B$ pathway components inhibitor of nuclear factor kappa $B$ kinase $(I k B)$ and reticuloendotheliosis oncogene A (RelA), as well as of MYD88 activator Bruton tyrosine kinase (BTK), suggesting that MYD88 may affect BTK activity. ST2825 effects were specific as MYD88-targeting siRNA also suppressed phosphorylation of $N F-k B$ signalling proteins and BTK in TMD8 cells. Conclusion: ST2825 may be a novel drug targeting not only B-lymphoid malignancies with MYD88 mutations, but also lymphoma and leukaemia with wild-type MYD88.
\end{abstract}

Myeloid differentiation primary response gene 88 (MYD88) is an adaptor protein that binds to toll-like receptors (TLR) and interleukin-1 receptor (IL1R) to form a homodimer which then activates the nuclear factor kappa B (NF-kB) pathway $(1,2)$. Activating MYD88 mutations constitutively induce NF-kB signalling, causing abnormal cell proliferation. MYD88 mutations are found in $90 \%$ of cases of lymphoplasmacytic lymphoma (Waldenström's macroglobulinemia) (3) and 30\% of activated B-cell type diffuse large B-cell lymphoma (DLBCL)

Correspondence to: Shuji Tohda, MD, Ph.D., Department of Laboratory Medicine, Tokyo Medical and Dental University, Yushima 1-5-45, Bunkyo-Ku, Tokyo 113-8519, Japan. Tel: +81 358035334, Fax: +81 358035629, e-mail: tohda.mlab@tmd.ac.jp

Key Words: MYD88, lymphoma, leukaemia, NF-kB.
(4), indicating MYD88 involvement in haematopoietic malignancies. However, the effects of MYD88-targeting drugs on the growth of lymphoma and leukaemia cells, especially cells with wild-type MYD88, have not been fully elucidated.

In this study, we investigated the role of MYD88 in stimulating the growth of lymphoma and leukaemia cells with and without MYD88 mutations and examined whether an MYD88 inhibitor could be used as novel drug-targeting molecular pathways involved in the progression. We also analysed the relationship between MYD88 and Bruton tyrosine kinase (BTK), which is a key molecule in B-cell receptor (BCR) signalling critical for abnormal growth of Bcell lymphoma cells (5).

\section{Materials and Methods}

Cell lines and treatment. Seven lymphoma and leukaemia cell lines were used. The TMD8 line was derived from DLBCL (6), Daudi from Burkitt lymphoma, NALM-6 from B-lymphoblastic leukaemia, Jurkat and KOPT-K1 from T-lymphoblastic leukaemia, and THP-1 and TMD7 from acute myeloid leukaemia. TMD8 cells carry a heterozygous MYD88 (L265P) mutation (7), whereas the other cell lines have wild-type MYD88 gene. TMD7 and TMD8 lines were established in our laboratory, Jurkat cells were purchased from the European Collection of Cell Cultures (Porton Down, Wiltshire, UK), and THP-1 cells were obtained from the Health Science Research Resource Bank (Osaka, Japan). The other cell lines were donated by Drs. Harashima and Orita, Fujisaki Cell Centre (Okayama, Japan). We also used normal lymphocytes isolated from two healthy volunteers who provided their informed consent to their use.

A synthetic peptidomimetic compound, ST2825, which inhibits MYD88 homodimerization (8), was purchased from ChemScene (Monmouth Junction, NJ, USA) and the BTK inhibitor ibrutinib (5) was obtained from SelleckChem (Houston, TX, USA). ST2825 and ibrutinib were dissolved in dimethyl sulfoxide (DMSO) at the concentration of $20 \mathrm{mM}$ and $10 \mathrm{mM}$, respectively, prior to use.

Cell growth assay. The colorimetric WST-8 assay (Dojindo Laboratories, Kumamoto, Japan) was used to evaluate cell growth. Cells were cultured in RPMI-1640 supplemented with $10 \%$ foetal bovine serum with or without inhibitors in 96-well culture plates in a humidified $5 \% \mathrm{CO}_{2}$ atmosphere. After $72 \mathrm{~h}$, WST-8 and 1methoxy-5-methylphenazinium methyl sulphate were added and the 


\section{Cell lines}

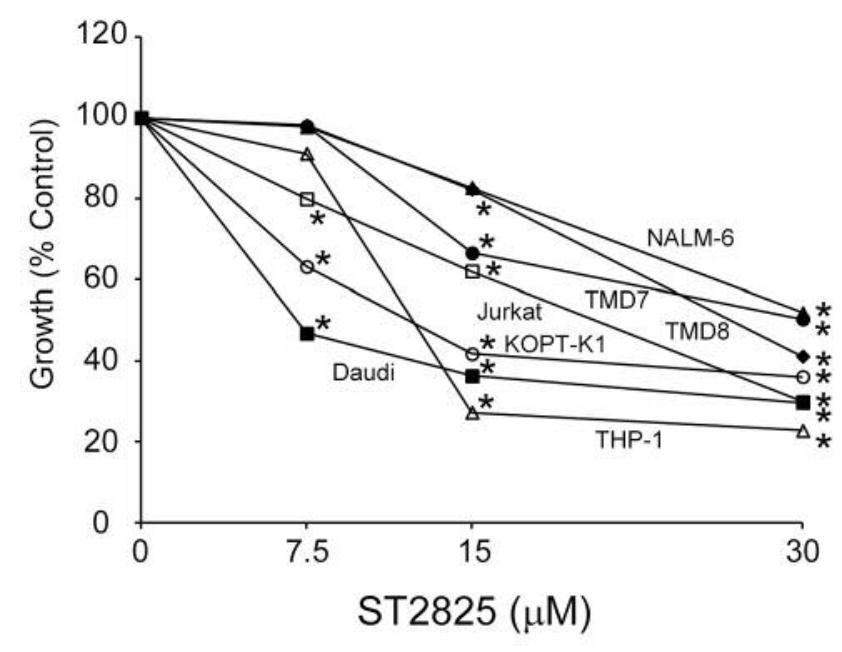

Lymphocytes

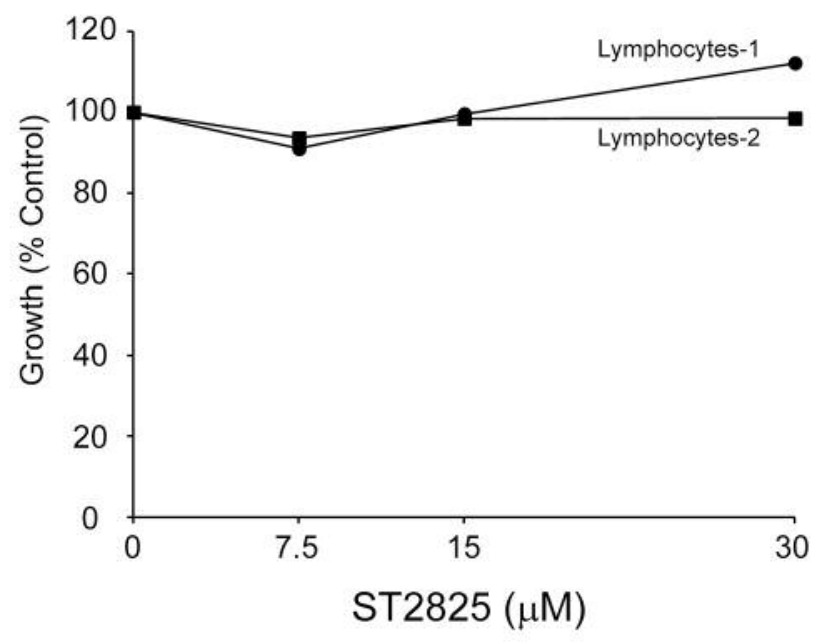

Figure 1. Effects of ST2825 on leukaemia/lymphoma cell growth. Cells were cultured with ST2825 for 3 days and evaluated for proliferation using a colorimetric assay. The results are expressed as the percentage of the mean optical density in ST2825-treated cells relative to that in control (dimethyl sulfoxide-treated) cells. Significantly different at $* p<0.05$ compared to control.

optical density (OD) was measured. Relative cell proliferation was calculated as the percentage of the mean OD value relative to that of untreated cells. The effects of inhibitors on cell morphology were examined in cytospin preparations stained with Wright's stain and observed under a microscope.

Apoptosis assay and cell-cycle analysis. Cells treated with ST2825 for $48 \mathrm{~h}$ were stained with propidium iodide (PI) to evaluate the cell cycle, and with annexin V-fluorescein isothiocyanate (FITC) and PI to examine apoptosis. The stained cells were then analysed by flow cytometry using a FACS Calibur cytometer (BD Biosciences, Franklin Lakes, NJ, USA).

MYD88 knockdown by siRNA. To confirm specificity of ST2825 effects, MYD88 was knocked-down by small interfering RNA (siRNA). Stealth RNAi ${ }^{\mathrm{TM}}$ pre-designed MYD88-specific siRNAs (HSS 181395, 181396, and 181397) and negative control Duplex were purchased from Life Technologies (Carlsbad, CA, USA). Cells were transfected with $80 \mathrm{nM}$ of each siRNA using the Neon ${ }^{\mathrm{TM}}$ pipette tip chamber-based electroporation system (Life Technologies) according to the manufacturer's instructions, and immediately transferred to culture medium.

Immunoblotting analysis of protein expression. ST2825-treated cells and siRNA-transfected cells were lysed and subjected to sodium dodecyl sulphate-polyacrylamide gel electrophoresis and immunoblotting with antibodies against MYD88, BTK, phosphoBTK, inhibitor of nuclear factor kappa B kinase (ІкB), phosphoІкB, reticuloendotheliosis oncogene A (RelA), phospho-RelA (Cell Signaling Technology, Danvers, MA, USA), and $\alpha$-tubulin (Abcam, Cambridge, MA, USA) used as a loading control. Immunoreactive bands were detected using an Enhanced Chemiluminescent Western Blotting kit (Pierce Biotechnology, Rockford, IL, USA). Each assay was performed at least twice to ascertain reproducibility.
Microarray analysis of mRNA expression. TMD8 cells were treated with $100 \mu \mathrm{M}$ ST2825 or DMSO (solvent control) for $6 \mathrm{~h}$ or transfected with $40 \mathrm{nM}$ MYD88 siRNA or control siRNA for $24 \mathrm{~h}$. Total RNA was extracted using the High Pure RNA isolation kit (Roche Diagnostics, Mannheim, Germany) and used to prepare cyanine-3-labelled cRNA which was hybridized to a SurePrint G3 Human GE microarray $8 \times 60 \mathrm{~K}$ v3 (Agilent Technologies, Santa Clara, CA, USA). The comprehensive gene-expression profile was analysed using the Agilent Feature Extraction 11.5.1.1 software.

\section{Results}

Effects of ST2825 on cell growth. Dose-response curves showed that ST2825 significantly suppressed the proliferation of all leukaemia/lymphoma cell lines starting at $15 \mu \mathrm{M}$ $(p<0.05)$, but did not affect the growth and viability of normal lymphocytes in the range of concentrations used (Figure 1).

Analysis of cytospin preparations showed that ST2825 induced apoptosis of all the studied cell lines, as evidenced by nuclear condensation and presence of apoptotic bodies (data not shown). Consistent with these results, flow cytometry revealed that in all cell lines, ST2825 increased the fraction of apoptotic (annexin-V-positive) cells (Figure 2). However, ST2825 treatment affected the cell cycle and induced $\mathrm{G}_{2} / \mathrm{M}$ arrest only in Daudi and Jurkat cells but not in TMD8 cells (Figure 3) or the other cell lines (data not shown).

Effects of ST2825 on signalling proteins. Protein expression analysis (Figure 4) revealed that MYD88 was expressed in all cell lines and that its levels were slightly reduced by treatment with $100 \mu \mathrm{M}$ ST2825 only in Daudi and KOPT-K1 cells. 


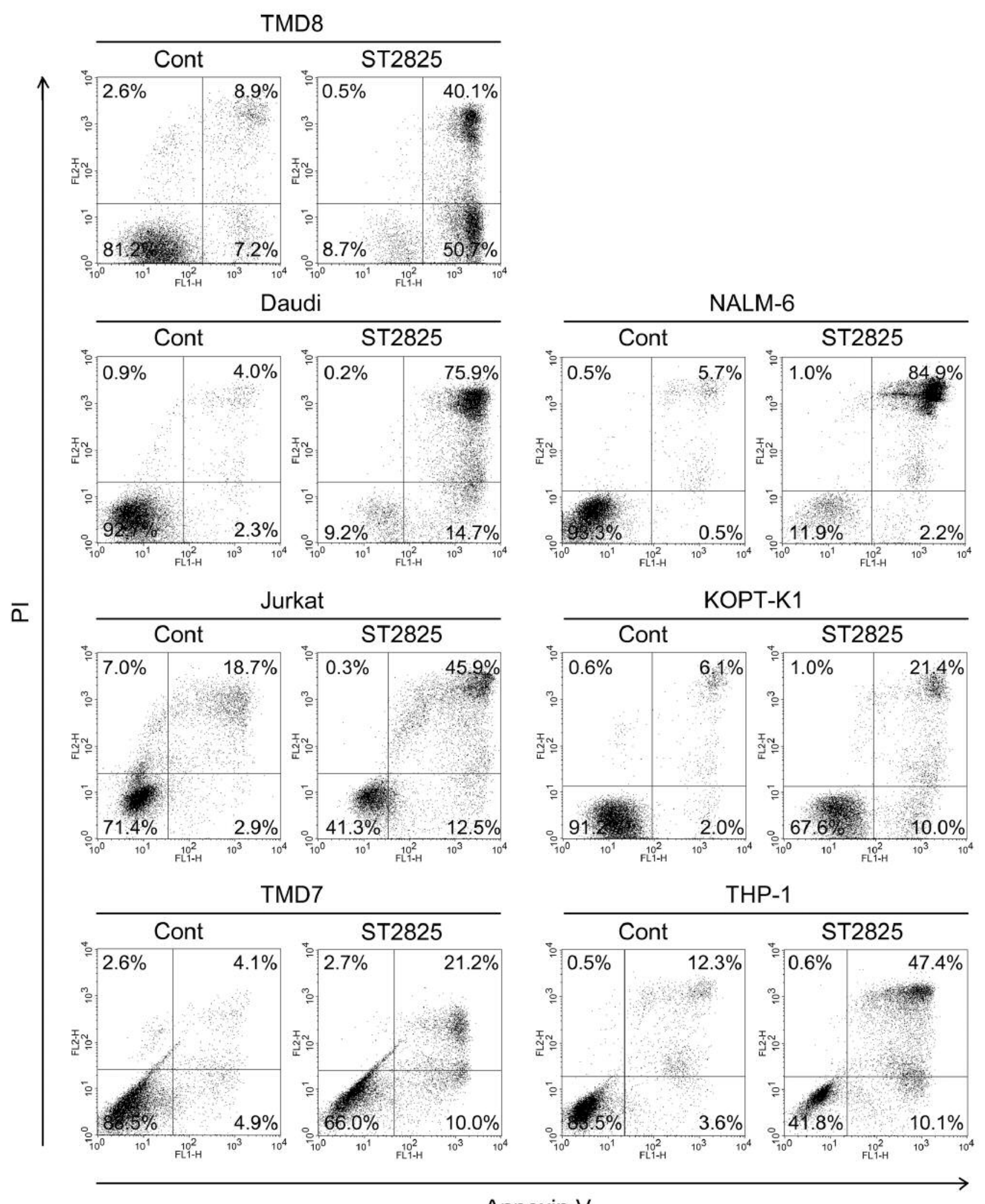

\section{Annexin V}

Figure 2. Apoptosis induction in leukaemia/lymphoma cells treated with ST2825. Cells were cultured with $30 \mu M$ ST2825 for 48 h, stained with annexin V-fluorescein isothiocyanate and propidium iodide (PI), and analysed for apoptosis by flow cytometry. 
TMD8
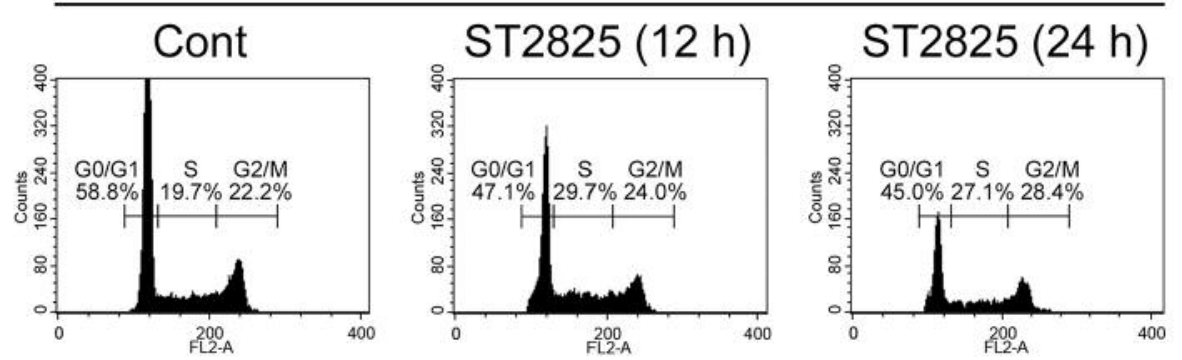

Daudi
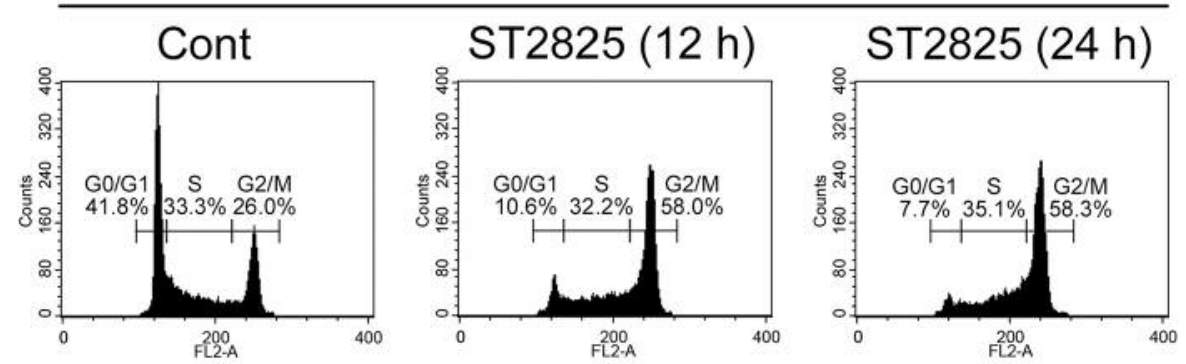

Jurkat
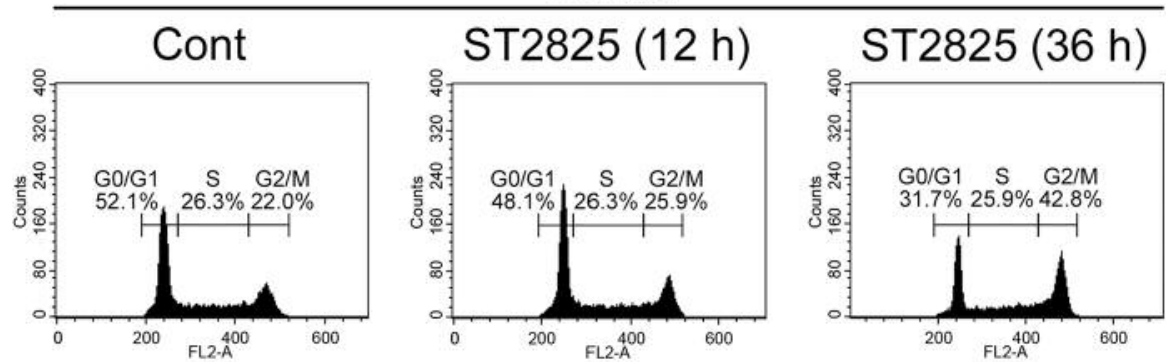

Figure 3. Cell-cycle changes in leukaemia/lymphoma cells treated with ST2825. Cells were cultured with $30 \mu M$ ST2825 for different durations, stained with propidium iodide, and analysed for cell-cycle progression by flow cytometry.

Among NF-kB signalling proteins, ST2825 down-regulated phosphorylation of IkB in TMD8, Daudi, KOPT-K1 and THP-1 cells, and reduced its expression in TMD8 and THP1 cells. At the same time, ST2825 inhibited phosphorylation of RelA in all cell lines without affecting its expression.

Interestingly, in TMD8, Daudi, and NALM-6 cells, ST2825 suppressed phosphorylation of BTK, which is known to act upstream of MYD88 (3), while the total BTK protein level was not affected.

Effects of MYD88 deficiency on signaling proteins. To ascertain whether the observed activities of ST2825 were due to the inhibition of MYD88 function and not to off-target effects, MYD88 was knocked-down using siRNA. As HSS 181395 (5'-GAGAUGGAUUUUGAGUACUUGGAGA-3') was the most potent among the three $M Y D 88$-specific siRNAs used, we analysed its effects on protein expression in TMD8 cells $48 \mathrm{~h}$ after transfection (Figure 5). The results indicate that MYD88 knockdown suppressed phosphorylation of IkB, RelA, and BTK, which is consistent with ST2825 effects (Figure 4).

Expression profiling by microarray. To further investigate the impact of MYD88 inhibition in leukaemia/lymphoma cells, we performed comprehensive analysis of gene expression. After selection of significantly expressed genes using the Agilent Feature Extraction software, we focused on NF-kB target genes $(9,10)$. Table I shows genes differentially expressed in ST2825-treated and MYD88 siRNA-transfected cells compared to respective controls. Among the NF-kB target genes, a change in expression of more than twofold was observed for myelocytomatosis oncogene $(M Y C)$ and interleukin10 (ILIO), as well as for interferon beta 1 (IFNBI) and B-cell leukemia/lymphoma 2 


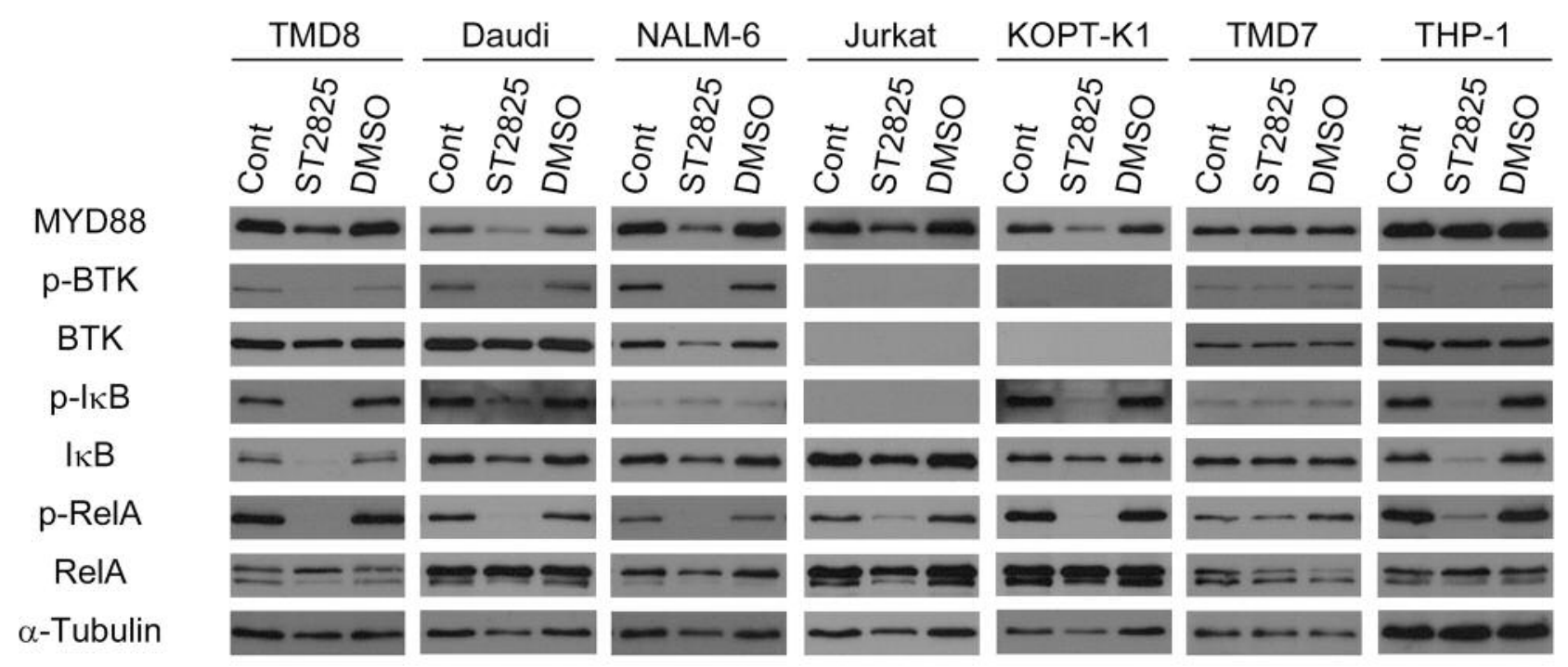

Figure 4. Effect of ST2825 on the expression of myeloid differentiation primary response gene 88 (MYD88) and nuclear factor kappa B (NF-kB) signaling proteins. Cells were cultured with $100 \mu \mathrm{M}$ ST2825 for $6 \mathrm{~h}$ and analysed for protein expression by immunoblotting. Dimethyl sulfoxide (DMSO) was used as a vehicle control and $\alpha$-tubulin as a loading control.

Table I. Microarray analysis of the effects of ST2825 and myeloid differentiation primary response gene 88 (MYD88) siRNA on the expression of nuclear factor kappa B (NF-kB) target genes. Log2 ratios of mRNA expression in ST2825-treated and MYD88 siRNA-transfected cells were normalized to those in dimethyl sulfoxide-treated and control siRNA-transfected cells, respectively.

\begin{tabular}{|c|c|c|c|}
\hline \multirow[t]{2}{*}{ Gene symbol } & \multirow[t]{2}{*}{ Encoded protein } & \multicolumn{2}{|c|}{$\log 2$ ratio mRNA expression } \\
\hline & & ST2825 & MYD88 siRNA \\
\hline$B C L 2$ & BCL2, apoptosis regulator & 0.630 & 0.449 \\
\hline CCND2 & Cyclin D2 & -0.551 & -0.148 \\
\hline$F A S$ & FAS cell surface death receptor & 0.505 & 0.194 \\
\hline FOS & FOS proto-oncogene, AP-1 transcription factor subunit & 1.862 & -2.534 \\
\hline HOXА9 & Homeobox A9 & -1.255 & -0.284 \\
\hline ICAMI & Intercellular adhesion molecule 1 & 0.480 & 0.362 \\
\hline IFNB1 & Interferon beta 1 & 0.817 & 0.461 \\
\hline$I F N G$ & Interferon gamma & 0.711 & -2.166 \\
\hline$I L 1 B$ & Interleukin 1 beta & -4.722 & -0.278 \\
\hline IL6 & Interleukin 6 & 0.903 & -0.939 \\
\hline IL10 & Interleukin 10 & -1.842 & -0.739 \\
\hline IRF1 & Interferon regulatory factor 1 & -0.485 & 0.421 \\
\hline$I R F 2$ & Interferon regulatory factor 2 & 0.394 & -0.280 \\
\hline IRF4 & Interferon regulatory factor 4 & 0.194 & -0.282 \\
\hline$J U N B$ & JUNB proto-oncogene, AP-1 transcription factor subunit & -0.512 & -0.294 \\
\hline$M Y C$ & MYC proto-oncogene, bHLH transcription factor & -0.749 & -0.391 \\
\hline$N F K B 1$ & Nuclear factor kappa B 1 & -0.454 & -0.126 \\
\hline$N F K B 2$ & Nuclear factor kappa B 2 & 0.003 & 0.156 \\
\hline NFKBIA & NFKB inhibitor alpha & -0.355 & 0.008 \\
\hline$R E L B$ & RELB proto-oncogene, NF-kB subunit & 0.312 & 0.236 \\
\hline STAT5A & Signal transducer and activator of transcription $5 \mathrm{~A}$ & 0.184 & -0.695 \\
\hline$T N F$ & Tumor necrosis factor & -1.148 & -0.301 \\
\hline
\end{tabular}




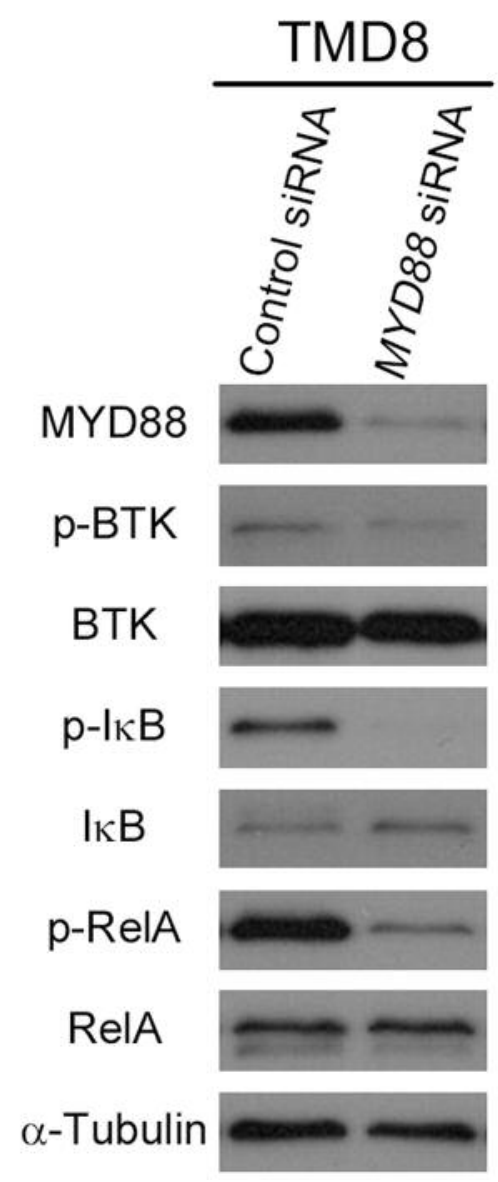

Figure 5. Effect of myeloid differentiation primary response gene 88 (MYD88) siRNA on the expression MYD88 and nuclear factor kappa $B$ $(N F-k B)$ signaling proteins in TMD8 cells. Cells were transfected with 80 nM MYD88 siRNA or control siRNA and analysed for protein expression after 48 h by immunoblotting.

(BCL2), which were down-regulated and up-regulated, respectively, by both ST2825 and MYD88 siRNA. On the other hand, FBJ osteosarcoma oncogene (FOS) was significantly up-regulated by ST2825, but down-regulated by MYD 88 siRNA.

Additive effects of ST2825 and ibrutinib on cell growth suppression. As MYD88 is a downstream target of BTK, we examined whether ST2825 might potentiate the effects of the BTK inhibitor ibrutinib on the growth of TMD8 cells. The results showed that a sub-optimal concentration of ibrutinib $(0.5 \mathrm{nM})$ suppressed proliferation of TMD8 cells, but its combination with $15 \mu \mathrm{M}$ ST2825 caused additive rather than synergistic effects on cell growth (Figure 6).

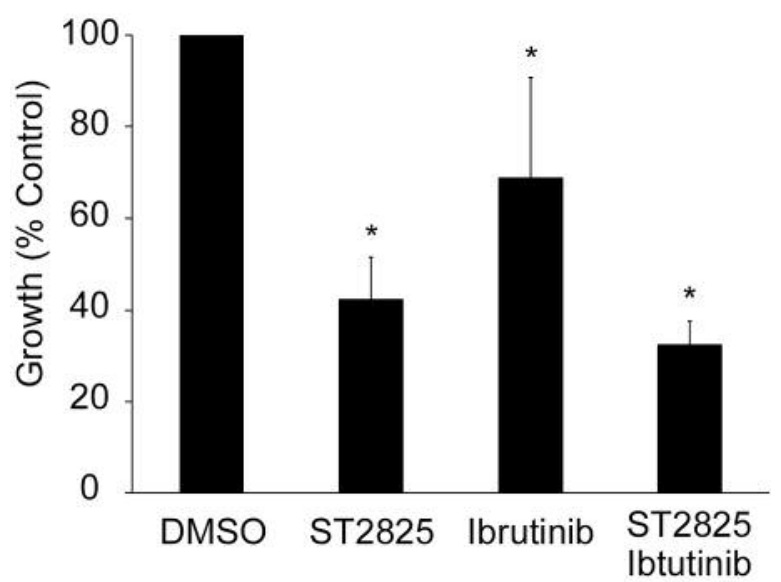

Figure 6. Additive effects of ST2825 on growth suppression of TMD8 cells by ibrutinib. Cells were cultured with $15 \mu \mathrm{M}$ ST2825, $0.5 \mathrm{nM}$ ibrutinib, or their combination for 3 days and evaluated for proliferation using a colorimetric assay. The results are expressed as the percentage of the mean optical density relative to that of control [dimethyl sulfoxide (DMSO)-treated] cells. Significantly different at $* p<0.05$ compared to DMSO control.

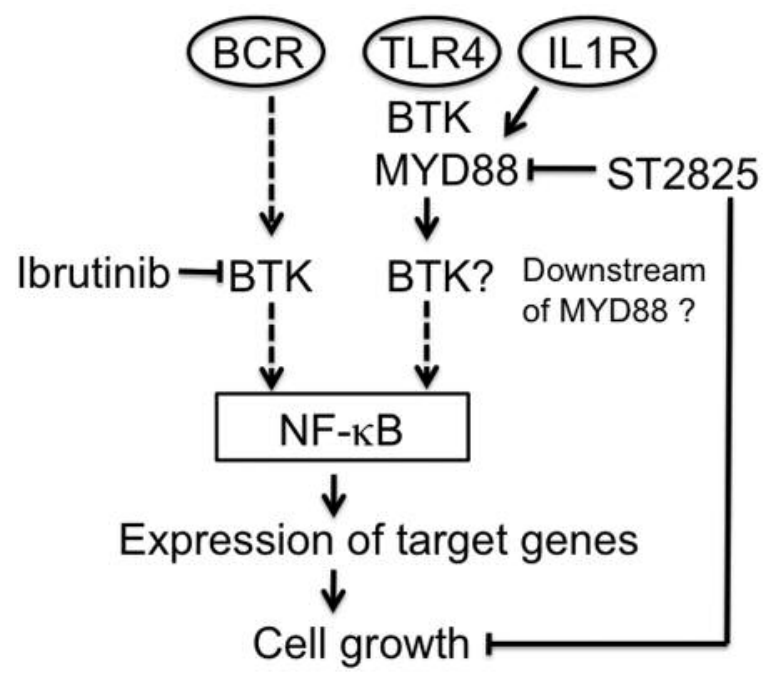

Figure 7. Schematic representation of ST2825 effects on myeloid differentiation primary response gene 88 (MYD88) and nuclear factor kappa $B(N F-k B)$ signalling. BCR: B-Cell receptor; BTK: Bruton tyrosine kinase; ILIR: interleukin 1 receptor; TLR4: toll-like receptor 4.

\section{Discussion}

We have shown here that the MYD88 inhibitor ST2825 suppressed the growth of various lymphoma and leukaemia cells and induced their apoptosis. It is known that abnormal proliferation of MYD88-mutated B-lymphoma cells is due 
to constitutive activation of MYD88-mediated signalling (4). Our findings suggest a possibility that MYD88 is also involved in the growth of T-lymphoid and myeloid leukaemia cells. We found that ST2825 treatment suppressed phosphorylation of NF-kB pathway components, suggesting that the inhibitory effects of ST2825 on cell growth may be due, at least in part, to down-regulation of NF-kB signalling.

Interestingly, we found that ST2825 also suppressed phosphorylation of BTK. In the TLR4-MYD88-NF-kB signalling pathway, the toll\&-interleukin-1 receptor (TIR) domain of TLR4 triggers MYD88 activation through interaction with TIR domain-containing adaptor protein (TIRAP) and BTK (3) located upstream of MYD88 (Figure 7). Our data suggest a possibility that BTK could also function downstream of MYD88 which may regulate BTK activity.

Furthermore, we found that $M Y D 88$ knockdown, similarly to ST2825, reduced phosphorylation of NF-kB pathway components and BTK, indicating that suppression of activation of these proteins was due to MYD88 inhibition and not to off-target effects of ST2825. However, such effects cannot be excluded as evidenced by the distinct influence of ST2825 and MYD88 siRNA on gene expression, suggesting that ST2825 may act also through MYD88independent mechanisms.

Our results indicate that ST2825 may be a novel drug for targeted therapy against MYD88-mutated B-lymphoid tumours, as well as other haematological malignancies not involving MYD88 mutations. It has also been reported that ST2825 suppressed in vitro growth of human hepatocellular carcinoma cells (11), which do not have MYD88 mutations. However, contrary to our expectations, ST2825 did not act synergistically with ibrutinib, clinically used for treatment of B-cell malignancies (12). Further investigation of the mechanisms underlying the effects of ST2825 is required.

\section{Acknowledgements}

This study was supported in part by a Grant-in-Aid for Scientific Research (C) from the Japan Society for the Promotion of Science (No. 26460669).

\section{References}

1 Akira $\mathrm{S}$ and Takeda K: Toll-like receptor signalling. Nat Rev Immunol 4: 499-511, 2004.

2 Warner N and Núñez G: MyD88: a critical adaptor protein in innate immunity signal transduction. J Immunol 190: 3-4, 2013.
3 Treon SP, Xu L, Yang G, Zhou Y, Liu X, Cao Y, Sheehy P, Manning RJ, Patterson CJ, Tripsas C, Arcaini L, Pinkus GS, Rodig SJ, Sohani AR, Harris NL, Laramie JM, Skifter DA, Lincoln SE and Hunter ZR: MYD88 L265P somatic mutation in Waldenström's macroglobulinemia. N Engl J Med 367: 826-833, 2012.

4 Ngo VN, Young RM, Schmitz R, Jhavar S, Xiao W, Lim KH, Kohlhammer H, Xu W, Yang Y, Zhao H, Shaffer AL, Romesser P, Wright G, Powell J, Rosenwald A, Muller-Hermelink HK, Ott G, Gascoyne RD, Connors JM, Rimsza LM, Campo E, Jaffe ES, Delabie J, Smeland EB, Fisher RI, Braziel RM, Tubbs RR, Cook JR, Weisenburger DD, Chan WC and Staudt LM: Oncogenically active MYD 88 mutations in human lymphoma. Nature 470: 115119, 2011

5 Davis RE, Ngo VN, Lenz G, Tolar P, Young RM, Romesser PB, Kohlhammer H, Lamy L, Zhao H, Yang Y, Xu W, Shaffer AL, Wright G, Xiao W, Powell J, Jiang JK, Thomas CJ, Rosenwald A, Ott G, Muller-Hermelink HK, Gascoyne RD, Connors JM, Johnson NA, Rimsza LM, Campo E, Jaffe ES, Wilson WH, Delabie J, Smeland EB, Fisher RI, Braziel RM, Tubbs RR, Cook JR, Weisenburger DD, Chan WC, Pierce SK and Staudt LM: Chronic active B-cell-receptor signaling in diffuse large B-cell lymphoma. Nature 463: 88-92, 2010.

6 Tohda S, Sato T, Kogoshi H, Fu L, Sakano S and Nara N: Establishment of a novel B-cell lymphoma cell line with suppressed growth by gamma-secretase inhibitors. Leuk Res 30 : 1385-1390, 2006.

7 Nogami S, Kawaguchi-Ihara N, Shiratori E, Ohtaka M, Itoh M and Tohda S: Detection of the MYD88 mutation by the combination of the allele-specific PCR and quenching probe methods. Int J Lab Hematol 39: 163-168, 2017.

8 Loiarro M, Capolunghi F, Fantò N, Gallo G, Campo S, Arseni B, Carsetti R, Carminati P, De Santis R, Ruggiero V and Sette C: Pivotal Advance: Inhibition of MYD88 dimerization and recruitment of IRAK1 and IRAK4 by a novel peptidomimetic compound. J Leukoc Biol 82: 801-810, 2007.

9 Pahl HL: Activators and target genes of Rel/NF-kB transcription factors. Oncogene 18: 6853-6866, 1999.

10 Smale ST: Hierarchies of NF-kB target-gene regulation. Nat Immunol 12: 689-694, 2011.

11 Deng Y, Sun J and Zhang LD: Effect of ST2825 on the proliferation and apoptosis of human hepatocellular carcinoma cells. Genet Mol Res 15: 15016826, 2016.

12 Gayko U, Fung M, Clow F, Sun S, Faust E, Price S, James D, Doyle M, Bari S and Zhuang SH: Development of the Bruton's tyrosine kinase inhibitor ibrutinib for B-cell malignancies. Ann NY Acad Sci 1358: 82-94, 2015 